\title{
Le infezioni catetere vascolare correlate: risultati di tre anni di sorveglianza (200 I-2003)
}

Vesselina Kroumova, Ilaria Crespi, Gian Lorenzo Molinari, Stefano Andreoni, Carmelina Di Natale, Paola Fonio, Giacomo Fortina

U.O.A. Laboratorio di Microbiologia e Virologia; Azienda Ospedaliera Maggiore della Carità di Novara

Key Words: CRI: catheter-related infection; CRBSI: catheter-related blood stream infection; catheter; infection control.

\section{SUMMARY}

Central venous catheter represents a major source of nosocomial bloodstream infection, which cause considerable excess morbidity. The diagnosis of catheter-related infections relies on the presence of clinical manifestation of infection and the evidence of colonization of the catheter tip by bacteria or fungi.

The most frequent pathogens were Gram-positive organisms, mainly coagulase negative staphylococci, followed by Gram-negative and mycetes.

During the years 200I-2003 we examined 2079 vascular catheters, of which 896 were positive for bacterial and fungal species. Of these, 675 (75,3\%) involved Gram-positive bacterial, I 45 (16,2\%) Gram-negative and 76 (8,5\%) mycetes.

\section{INTRODUZIONE}

Le infezioni ospedaliere sono acquisite durante il ricovero in ospedale. Esse possono manifestare segni clinici durante il periodo di ricovero o, in alcuni casi, anche dopo che il paziente è stato dimesso a condizione che le stesse siano state contratte durante la degenza ospedaliera. Le infezioni ospedaliere, per definizione, devono insorgere dopo 48 ore dall'ingresso in Ospedale e, conseguentemente, non devono essere né manifeste clinicamente, né in incubazione al momento del ricovero (8).

Responsabili di queste infezioni sono batteri, virus, parassiti e miceti.

Tali microrganismi possono essere acquisiti in vario modo sia come infezione endogena provocata dalla normale popolazione microbica presente nell'individuo che in particolari condizioni può determinare infezioni, sia come infezione crociata esogena provocata da flora proveniente da altri individui, operatori sanitari compresi e sia come infezione ambientale esogena endemica o epidemica provocata da microrganismi che sono in grado di sopravvivere nell'ambiente (14).

Una delle infezioni nosocomiali più frequenti e più importanti per $\mathrm{i}$ risvolti clinici è quella da catetere vascolare (catheter-related-infection: CRI) (6).

Questa infezione, in particolare quando associata a batteriemia (CRBSI: catheter-related bloodstream infection), è la principale complicanza del posizionamento di un catetere vascolare (13).

Negli U.S.A la C.R.I. è presente nel 3-7\% dei pazienti con catetere venoso centrale e in circa $1 ' 1 \%$ di quelli con catetere arterioso $(12,13)$.

L'uso di cateteri vascolari è una delle novità prin- cipali della moderna medicina (4). Questa pratica risulta molto diffusa in alcuni pazienti come ad esempio i dializzati dove oltre il $18,9 \%$ è portatore di catetere e spesso anche per lunghi periodi (5). Le più importanti complicanze dell'uso di questi device sono l'insorgenza di infezioni che mostrano una significativa mortalità (19). L'elevata incidenza di infezioni associate alla presenza di cateteri intravascolari è spesso dovuta alla manualità nel posizionarli e nel gestirli. Tutto questo ha portato a definire procedure certe per ridurre l'insorgenza di queste infezioni (18).

Il Centers for Disease Control and Prevention definisce come colonizzazione di un catetere la presenza di almeno 15 CFU ottenute da una coltura semiquantitativa o di $>1000 \mathrm{CFU}$ ottenute da una coltura quantitativa dal frammento prossimale o distale del catetere $(2,15)$. Un'infezione ematica correlata al catetere (Catheter-related bloodstream infection) (CRBSI) viene definita quando viene isolato il medesimo microrganismo dall'emocoltura e dalla coltura del catetere in presenza di segni clinici di infezione senza apparenti altri focolai di possibile infezione $(4,15)$.

Il catetere può essere colonizzato per migrazione del microrganismo direttamente dalla pelle nel momento di inserzione del catetere oppure attraverso il giunto del sistema di infusione (11). Un'altra possibilità, anche se sembra di minor importanza nel determinare fatti settici, può essere la via ematogena o l'utilizzo di infusioni contaminate $(11,15,18)$. Numerosi sono stati gli studi relativi alle cause che sottendono a queste infezioni. Secondo Jaber (10) tre sono i principali fattori che sembrano maggiormente responsabili del fenomeno: le difese dell'ospite, la virulenza del 
microrganismo e le manovre chirurgiche che portano all'inserimento del device.

L'adesione del microrganismo alla superficie del catetere è un passaggio critico del processo di colonizzazione batterica $(9,17)$. Le diminuite difese immunitarie, in particolare del sistema complemento e dei polimorfonucleati, rivestono un importante ruolo nel favorire l'adesione iniziale e l'irreversibilità di questo processo, passaggio fondamentale per giungere alla colonizzazione del device (20). Vi è da notare inoltre che la presenza del biofilm prodotto dalle cellule batteriche è responsabile di un'aumentata resistenza dei microrganismi nei confronti degli antibiotici. Il meccanismo responsabile di questo fenomeno è probabilmente almeno duplice. Sembra Infatti concorrere a questa aumentata resistenza sia la presenza del glicocalice che blocca la penetrazione di alcune molecole di antibiotico, sia un metabolismo più lento della cellula batterica che la rende meno sensibile all' azione dell'antimicrobico (1). Considerando quanto già sia delicata la terapia antibiotica in ambiente ospedaliero dove vengono inevitabilmente selezionati ceppi multiresistenti vediamo come, anche questo fenomeno, possa essere di particolare importanza nell'evoluzione dell'infezione correlata a catetere vascolare.

L'obiettivo di questo studio retrospettivo è determinare l'incidenza delle infezioni catetere correlate, i microrganismi coinvolti e rilevare la loro antibiotico sensibilità nel periodo dei tre anni esaminati.

\section{MATERIALI E METODI}

Per la ricerca dei microrganismi nei cateteri vascolari è stato utilizzato il metodo semiquantitativo che consiste nel far ruotare la punta del catetere sulla superficie di una piastra di terreno colturale. La soglia di positività corrisponde a $\geq 15$ unità formanti colonie $(2,15)$.

I terreni colturali utilizzati sono stati Columbia 5\% Becton Dickinson, G.C. Choco Becton Dickinson, Sabouraud Agar Becton Dickinson.

Le Emocolture sono state effettuate utilizzando i flaconi "BACTEC" forniti dalla ditta Becton Dickinson e per il loro utilizzo sono state seguite le indicazioni fornite dalla ditta.

L'identificazione della specie è stata praticata secondo le metodiche tradizionali utilizzando i sistemi commerciali Vitek2 e Api-System della ditta bioMèrieux. La sensibilità nei confronti delle varie molecole di antibiotici è stata saggiata utilizzando il sistema automatico Vitek2 della ditta bioMèrieux. L'indagine epidemiologica è stata eseguita utilizzando il sistema LMX della ditta Bayer ed il programma informatico MERCURIO della ditta Dianoema.
Per i controlli di qualità sono stati impiegati Escherichia coli ATCC 35150, Pseudomonas aeruginosa ATCC 27853, Staphylococcus aureus ATCC 8325.

\section{RISULTATI E DISCUSSIONE}

Nel corso delle annate 2001-2003 furono sottoposti a controllo microbiologico 2079 cateteri vascolari, con una distribuzione nei tre anni di 521,853 e 705 colture. Nella nostra analisi per ogni singolo paziente è stato considerato solo il primo campione positivo. Utilizzando questo metodo il numero di campioni positivi è risultato essere nel primo anno di 266 , pari al $48,0 \%$, di 384 pari al 51,5\% nel secondo e di 246 pari al $50,6 \%$ nel terzo per un totale di 896 microrganismi identificati con una percentuale di positività complessiva pari al $50,2 \%$.

Nella tabella 1 sono riportati tutti i microrganismi isolati nel corso dell'indagine con le percentuali dei singoli anni e quella complessiva.

Degli 896 stipiti isolati (figura I), 675 pari al $75,3 \%$ corrisposero a batteri Gram-positivi, con una percentuale che è passata dal $66,9 \%$ dell'anno 2001 al 78\% del 2003. I batteri Gram-negativi isolati nei tre anni in totale furono 145 pari al $16,2 \%$. Per questi microrganismi si evidenzia una significativa riduzione nella percentuale di positività dopo il primo anno. L'isolamento di miceti risultò essere complessivamente di 76 ceppi pari all' $8,5 \%$ con una distribuzione sufficientemente costante nei tre anni.

Nell'ambito dei 675 batteri Gram-positivi è risultato nettamente predominante il genere Staphylococcus con 577 isolamenti, ed una percentuale di positività via via crescente (figura II). In particolare, S. epidermidis, con i suoi 421 stipiti ed una frequenza che nei tre anni passa dal $33,5 \%$ al 56,9\% risultò in assoluto il microrganismo più frequentemente isolato, mentre $S$. aureus, con i suoi 82 stipiti, mostrò una percentuale di positività in diminuzione passando dall' $11,6 \% \mathrm{al}$ $5,7 \%$ nel corso dei tre anni. Significativa anche la presenza di batteri del genere Streptococcus benché essi, come anche i batteri del genere Enterococcus, risultino in costante diminuzione nel corso del triennio.

Nell'ambito dei 145 batteri Gram-negativi (figura III) vi è una prevalenza di enterobatteri. Per questi microrganismi si evidenzia una percentuale in continua diminuzione così come per i non fermentanti che dimezzano praticamente la loro percentuale di isolamento. Diverso il comportamento dei miceti. Infatti a fronte di una diminuzione tra il primo e il secondo anno con un passaggio dall' $11,3 \%$ al $6,5 \%$, si evidenzia un successivo aumento nel corso dell'ultimo anno dove la per- 
centuale risale all' $8,6 \%$.

Può essere interessante paragonare la nostra casistica, anche in considerazione delle dimensioni della struttura ospedaliera a cui fa riferimento, alla nota pubblicata da Paragioudaky (16). Infatti le due strutture ospedaliere sono simili come numero di posti letti e come distribuzione di reparti. Anche il numero di campioni esaminati è simile considerando che la casistica greca si articola su due anni mentre la nostra su tre. Per ciò che riguarda lo $S$. epidermidis, benchè il lavoro di Paragioudaky non differenzi i vari coagulasi negativi, abbiamo una quasi sovrapposizione di dati che ritroviamo anche nei miceti. Per tutti gli altri microrganismi troviamo invece differenze significative tra i due lavori probabilmente legati alla popolazione batterica residente di ciascuna realtà e questo indica quanto gli studi epidemiologici abbiano importanza per un quadro generale ma non possono prescindere da una conoscenza precisa di quella che è la realtà locale.

Per quanto riguarda l'antibiotico resistenza abbiamo voluto considerare solo quella relativa allo $S$. epidermidis in quanto lo stesso risulta il microrganismo di gran lunga più frequentemente isolato nella nostra casistica e conseguentemente con dati non inficiati da eventuali scarsi isolamenti di un singolo anno. Nella figura IV sono presentate le percentuali di resistenza nei confronti di alcune molecole di antibiotico. Un primo dato sicuramente importante è la costanza delle resistenze nell'arco dei tre anni ad eccezione del gruppo fluorochinolonico dove è evidente un importante incremento dal 2001 al 2002 (14 punti percentuali): situazione che poi è stata confermata anche nel 2003. Questo dato è probabilmente legato all'aumentato utilizzo di molecole fluorochinoloniche che si è verificato nel nostro Ospedale a partire dal 2002. Per le altre molecole, come era prevedibile attendersi, la maggior resistenza si ritrova nei confronti di oxacillina dove è anche presente un incremento, seppur minore rispetto ai fluorochinolonici, ma pur sempre di 6 punti percentuali nell'ultimo anno. Questo dato in particolare conferma, purtroppo, un trend di crescita nella resistenza dell'oxacillina nei confronti degli S. epidermidis in particolare e degli Stafilococchi in generale (3).

Relativamente ai dati della farmacoresistenza possiamo segnalare che anche in questo caso le resistenze nei confronti di oxacillina e di tutte le altre molecole beta lattamiche mostrano una sovrapposizione con il lavoro di Paragioudaky (16). Anche la resistenza nei confronti degli aminoglicosidi è praticamente sovrapponibile come pure quella nei confronti di Vancomicina. Le resistenze da loro evidenziate nei confronti dei fluo- rochinolonici sono simili a quelle da noi ritrovata nel primo anno. Considerando che il lavoro greco si riferisce agli anni 1999 e 2000 potrebbe essere suggestivo ipotizzare anche per la loro realtà un innalzamento della resistenza come si è verificato da noi. Questo potrebbe essere confermato anche in considerazione del fatto che i nostri dati sono simili a quelli presentati dal Dr. Gesu nell' indagine policentrica sull'attività in vitro verso Levofloxacina e Ciprofloxacina (7).

Notevolmente diversa appare la resistenza ritrovata nei confronti di Trimethoprim/Sulfametoxazolo che presenta, nella nostra casistica, una percentuale notevolmente più bassa.

Infine si è voluto indagare quante di queste positività corrispondessero a situazioni cliniche gravi. Essendo improponibile effettuare un'indagine retrospettiva su dati clinici si è pensato di ricercare la contemporanea presenza di positività all'emocoltura per lo stesso microrganismo associato ad un identico profilo di antibiotipia. Infatti sia la richiesta di effettuare un indagine come l'emolcoltura sia la sua positività è spesso segno di un quadro clinico importante e non di una semplice colonizzazione del catetere. Per questo vengono considerati tutti quei pazienti che presentavano una contemporanea positività oltre che per il catetere vascolare anche per l'emocoltura e fra questi quelli con positività per lo stesso microrganismo. Ulteriormente si è voluto, in mancanza di una possibile indagine molecolare retrospettiva, ricercare anche l'identicità nelle resistenze antibatteriche saggiate (antibiotipia). La ricerca ha riguardato i microrganismi più frequentemente isolati non avendo significato un'eventuale concordanza o meno su singoli casi. Sono stati presi in considerazione i batteri del genere $S$. epidermidis, $S$. aureus, Enterobacter cloacae, E. coli, P. aeruginosa e Candida albicans per un totale di 621 microrganismi pari all' $69,3 \%$ del totale. Di questi 78 , con una percentuale complessiva pari al $12,6 \%$, sono risultati positivi contemporaneamente alle indagini catere-emocoltura mostrando inoltre un identico profilo nei confronti degli antibiotici saggiati (tabella 2).

L'analisi di questi dati mostra che all'interno del genere Staphyloccoccus esite un'importante differenza fra lo $S$. aureus e lo $S$. epidermidis. Infatti a fronte di un rapporto di positività 1 a 5 fra questi due microrganismi nelle colture da catetere il rapporto scende a 1 a 2 nelle emocolture. Sorprendente è pure il dato relativo ai Gram negativi dove si evidenzia una percentuale molto diversa, perlomeno nella nostra casistica, fra l'E. cloacae e lo $P$. aeruginosa. Infatti nel primo caso più di un terzo delle positività da catetere sono accompagnate da isolamento del medesimo 
microrganismo nell'emocoltura. Al contrario in nessun caso è stata riscontrata la positività nell'emocoltura per quei pazienti che risultavano positivi per $P$. aeruginosa nella coltura da catetere. Questo dato non è sicuramente facilmente interpretabile e richiede certamente ulteriori e più approfondite analisi come pure è da valutare con molta attenzione l'elevata percentuale con cui $C$. albicans è contemporaneamente positiva nelle emocolture. Indagini relative agli stretti rapporti fra microrganismo e device e in particolare la ricerca di determinanti genetici che possano con- sentire l'espressione di meccanismi di adesione al catetere e di una capacità autonoma di moltiplicazione su tale materiale potranno in un futuro meglio spiegare tali differenze. Come risulterà indispensabile un'analisi della identicità dei due microrganismi isolati che possa essere supportata da elementi certi rispetto a quelli qui osservati come, ad esempio una tipizzazione molecolare. Visti i risultati ottenuti da questa prima indagine epidemiologica è proprio in questa direzione che cercheremo di sviluppare i futuri approfondimenti su questa materia.

Tabella I. Incidenza di microrganismi isolati da cateteri venosi centrali anni: 200 I-2003

\begin{tabular}{|c|c|c|c|c|c|c|}
\hline \multirow{2}{*}{$\begin{array}{l}\text { Microrganismo } \\
\text { Achromobacter xylosoxidans ssp } \\
\text { denitrificans }\end{array}$} & \multicolumn{2}{|c|}{2001 - anno } & \multicolumn{2}{|c|}{2002 - anno } & \multicolumn{2}{|c|}{2003 - anno } \\
\hline & & & & & 1 & $(0,41)$ \\
\hline Acinetobacter baumannii & & & 10 & $(2,60)$ & & \\
\hline Acinetobacter lwoffii/alcaligenes & 2 & $(0,75)$ & 2 & $(0,52)$ & 1 & $(0,41)$ \\
\hline Agrobacterium tumefaciens & & & 2 & $(0,52)$ & & \\
\hline Alcaligenes faecalis & 1 & $(0,38)$ & & & & 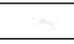 \\
\hline Alcaligenes species & 1 & $(0,38)$ & & & & \\
\hline Bacillus pumilus & 1 & $(0,38)$ & & & & \\
\hline Bacillus species & 5 & $(1,88)$ & 9 & $(2,34)$ & 1 & $(0,41)$ \\
\hline Bacillus subtilis & 1 & $(0,38)$ & & & & \\
\hline Candida albicans & 15 & $(5,64)$ & 20 & $(5,21)$ & 10 & $(4,07)$ \\
\hline Candida glabrata & 3 & $(1,13)$ & & & 3 & $(1,22)$ \\
\hline Candida parapsilosis & 8 & $(3,01)$ & 3 & $(0,78)$ & 5 & $(2,03)$ \\
\hline Candida species & 2 & $(0,75)$ & 1 & $(0,26)$ & & \\
\hline Candida tropicalis & 1 & $(0,38)$ & & & 2 & $(0,81)$ \\
\hline Citrobacter freundii & 3 & $(1,13)$ & 1 & $(0,26)$ & 1 & $(0,41)$ \\
\hline Clavispora lusitaniae & & & & & 1 & $(0,41)$ \\
\hline Corynebacterium bovis & 1 & $(0,38)$ & & & & \\
\hline Corynebacterium jeikeium & & & 2 & $(0,52)$ & 2 & $(0,81)$ \\
\hline Corynebacterium striatum & 2 & $(0,75)$ & & & 2 & $(0,81)$ \\
\hline Corynebacterium xerosis & & & 3 & $(0,78)$ & 1 & $(0,41)$ \\
\hline Enterobacter aerogenes & 2 & $(0,75)$ & 3 & $(0,78)$ & 2 & $(0,81)$ \\
\hline Enterobacter cloacae & 4 & $(1,50)$ & 12 & $(3,13)$ & 6 & $(2,44)$ \\
\hline Enterococcus durans & 1 & $(0,38)$ & & & & \\
\hline Enterococcus faecalis & 7 & $(2,63)$ & 15 & $(3,91)$ & 3 & $(1,22)$ \\
\hline Enterococcus faecium & 3 & $(1,13)$ & & & & \\
\hline Escherichia coli & 14 & $(5,26)$ & 8 & $(2,08)$ & 1 & $(0,41)$ \\
\hline $\begin{array}{l}\text { Issatchenkia orientalis (Candida } \\
\text { krusei) }\end{array}$ & 1 & $(0,38)$ & & & & \\
\hline Klebsiella oxytoca & 2 & $(0,75)$ & & & 1 & $(0,41)$ \\
\hline Klebsiella pneumoniae & 4 & $(1,50)$ & 3 & $(0,78)$ & 3 & $(1,22)$ \\
\hline $\begin{array}{l}\text { Klebsiella pneumoniae ssp } \\
\text { rhinoscleromatis }\end{array}$ & 1 & $(0,38)$ & & & & \\
\hline Kocuria rosea & & & 1 & $(0,26)$ & 2 & $(0,81)$ \\
\hline Kocuria varians & & & & & 1 & $(0,41)$ \\
\hline Micrococcus luteus & & & 3 & $(0,78)$ & 3 & $(1,22)$ \\
\hline Micrococcus species & 1 & $(0,38)$ & & & & \\
\hline Moraxella osloensis & 2 & $(0,75)$ & & & & \\
\hline Morganella morganii & & & 1 & $(0,26)$ & 1 & $(0,41)$ \\
\hline Mucor species & & & 1 & $(0,26)$ & & \\
\hline $\begin{array}{l}\text { Neisseria subflava } \\
\end{array}$ & 1 & $(0,38)$ & & & & \\
\hline Oerskovia species & 1 & $(0,38)$ & & & & \\
\hline Peptostreptococcus anaerobius & & & & & 1 & $(0,41)$ \\
\hline Pichia etchellsii & 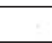 & & 2 & $(0,52)$ & & \\
\hline Proteus mirabilis & 1 & $(0,38)$ & 2 & $(0,52)$ & 6 & $(2,44)$ \\
\hline Pseudomonas aeruginosa & 10 & $(3,76)$ & 8 & $(2,08)$ & 10 & $(4,07)$ \\
\hline Pseudomonas fluorescens & & & 2 & $(0,52)$ & & \\
\hline Pseudomonas species & 5 & $(1,88)$ & 1 & $(0,26)$ & & \\
\hline Ralstonia pickettii & 1 & $(0,38)$ & & & & \\
\hline
\end{tabular}


Segue:

\begin{tabular}{lrrrrrr}
\hline Microrganismo & 2001 - anno & 2002 - anno & \multicolumn{2}{c}{ 2003 - anno } \\
\hline Raoultella ornithinolytica & 1 & $(0,38)$ & & & & \\
\hline Rhodotorula mucilaginosa & 1 & $(0,38)$ & & & & \\
\hline Serratia marcescens & 1 & $(0,38)$ & & & & \\
\hline Sphingomonas paucimobilis & 1 & $(0,38)$ & & & & \\
\hline Staphylococcus aureus & 31 & $(11,65)$ & 37 & $(9,64)$ & 14 & $(5,69)$ \\
\hline Staphylococcus auricularis & 1 & $(0,38)$ & & & 2 & $(0,81)$ \\
\hline Staphylococcus capitis & 5 & $(1,88)$ & 4 & $(1,04)$ & 3 & $(1,22)$ \\
\hline Staphylococcus chromogenes & & & 2 & $(0,52)$ & 1 & $(0,41)$ \\
\hline Staphylococcus chromogenes/hyicus & & & & & 1 & $(0,41)$ \\
\hline Staphylococcus cohnii & & & 2 & $(0,52)$ & 1 & $(0,41)$ \\
\hline Staphylococcus epidermidis & 89 & $(33,46)$ & 192 & $(50,00)$ & 140 & $(56,91)$ \\
\hline Staphylococcus haemolyticus & 4 & $(1,50)$ & 9 & $(2,34)$ & 1 & $(0,41)$ \\
\hline Staphylococcus hominis & 10 & $(3,76)$ & 5 & $(1,30)$ & 2 & $(0,81)$ \\
\hline Staphylococcus intermedius & & & 1 & $(0,26)$ & & \\
\hline Staphylococcus schleiferi & & & 2 & $(0,52)$ & 1 & $(0,41)$ \\
\hline Staphylococcus simulans & & & 2 & $(0,52)$ & & \\
\hline Staphylococcus warneri & & & 4 & $(1,04)$ & 4 & $(1,63)$ \\
\hline Staphylococcus xylosus & 2 & $(0,75)$ & & & 2 & $(0,81)$ \\
\hline Streptococcus agalactiae & & & 1 & $(0,26)$ & & \\
\hline Streptococcus intermedius/milleri & 3 & $(1,13)$ & 2 & $(0,52)$ & & \\
\hline Streptococcus mitis & 5 & $(1,88)$ & 3 & $(0,78)$ & 2 & $(0,81)$ \\
\hline Streptococcus pyogenes & & & & & 1 & $(0,41)$ \\
\hline Streptococcus sanguis i & & & & & 1 & $(0,41)$ \\
\hline Streptococcus viridans & 5 & $(1,88)$ & 3 & $(0,78)$ & & \\
\hline TOTALE GLOBALE & $\mathbf{2 6 6}$ & & 384 & & $\mathbf{2 4 6}$ & \\
\hline
\end{tabular}

Tabella 2. Contemporanea positività per catetere ed emocoltura di microrganismi con identico antibiogramma

\begin{tabular}{lccc}
\hline MICRORGANISMO & CATETERI & EMO & PERCENTUALE \\
\hline S.epidermidis & 421 & 32 & 7,6 \\
\hline S.aureus & 82 & 15 & 18,3 \\
\hline E.cloacae & 22 & 8 & 36,4 \\
\hline E.coli & 23 & 3 & 13,0 \\
\hline P.aeruginosa & 28 & 0 & 0 \\
\hline C.albicans & 45 & 20 & 44,4 \\
\hline
\end{tabular}

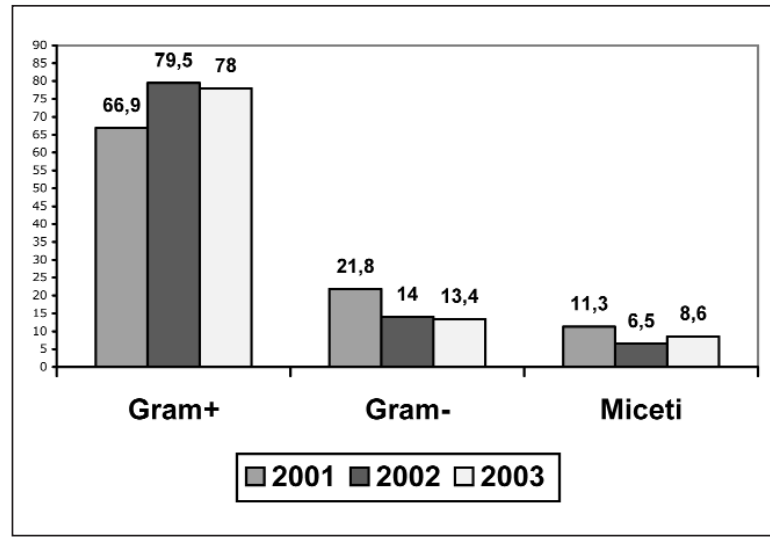

Figura I. Percentuale di positività dei microrganismi isolati negli anni 200I-2003

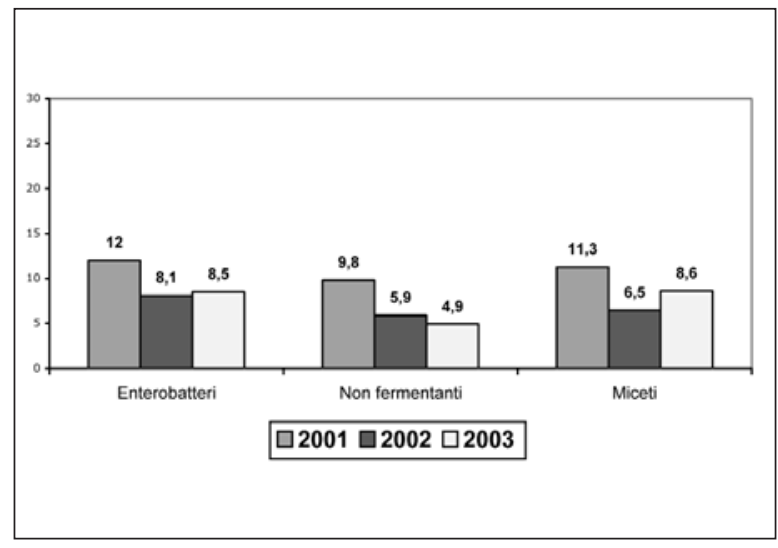

Figura III. Percentuale di positività di microrganismi Gram (-) e miceti isolati negli anni 200I-2003

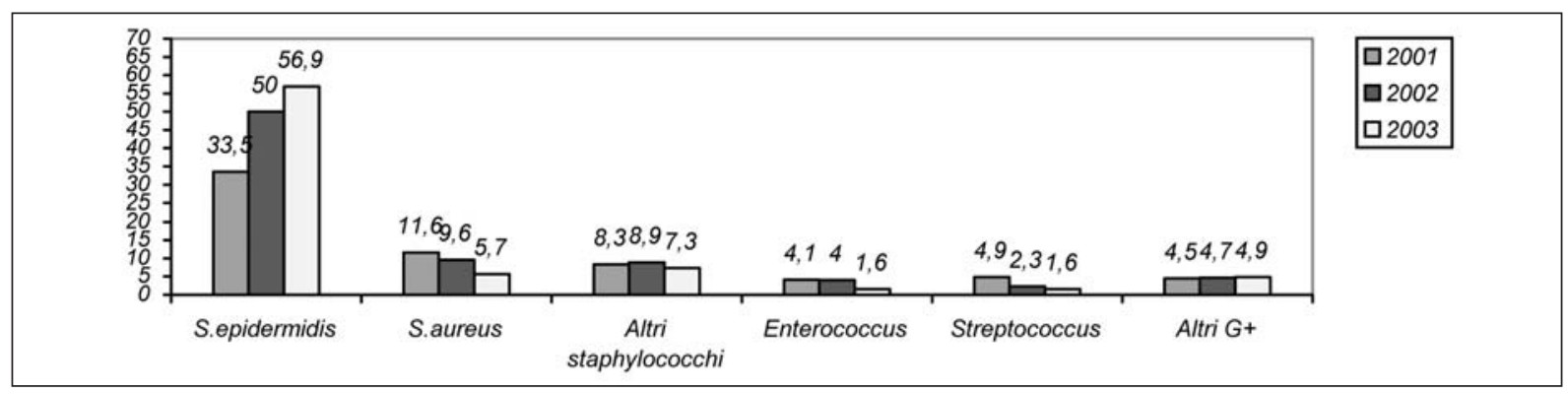

Figura II. Percentuale di positività dei microrganismi Gram +isolati negli anni 200 I-2003 


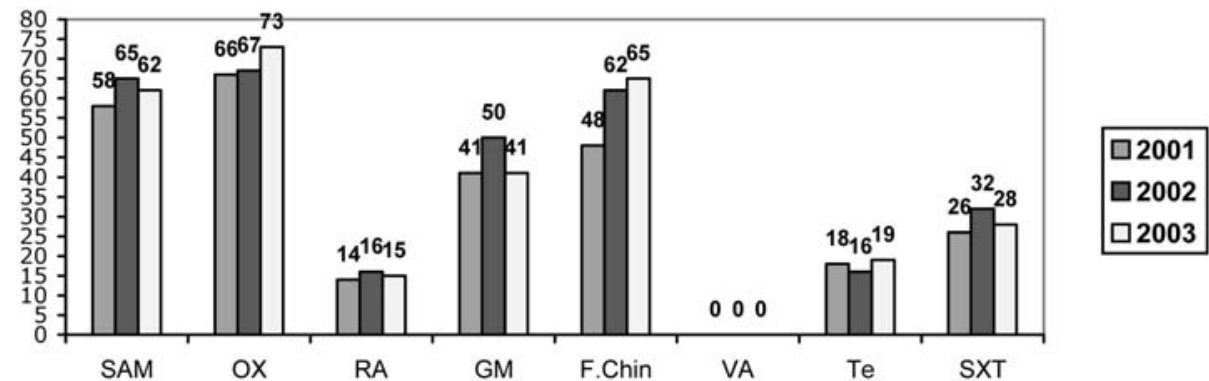

SAM:Ampicillina-sulbactam; OX: Oxacillina; RA: Rifampicina; GM: Gentamicina; MA: Cefamandolo;

F. Chin. Fluorchinolonici; VA: Vancomicina; Te: Tetracicline; SXT: Trimethoprim/ Sulfametoxazolo.

Figura IV. Antibioticoresistenza di S. epidermidis negli anni 200I-2003

\section{BIBLIOGRAFIA}

1. Anderl JN, Franklin MJ, Stewart PS. Role of antibiotic penetration limitation in Klebsiella pneumonite biofilm resistance to ampicillin and ciprofloxacin. Antimicrob Agents Chemother. 2000, 44, 1818-24.

2. Capdevila JA, Planes AM, Palomar M, et al. Value of differential quantitative blood cultures in tha diagnosis of cathater-related sepsis. Eur J Clin Micro Infect Dis 1992; 11: 403-7.

3. Carratalà J. Role of antibiotic prophylaxis for the prevention of intravascular catheter-related infections Clin Microbiol Infect 2001; 7 Suppl.4: 83-90.

4. Crump JA, Collingnon PJ. Intravascular catheterassociated infections. Eur Jclin Microbiol Infect Di. 2000; 19: 1-8.

5. Develter W, De Cubber A, Van Biesen W, Vanholder R, Lameire N. Survival and complications of indwelling venous catheter for permanent use in hemodialysis patients. Artif Organs 2005; 29: 399-405.

6. Emory TG, Gaynes RP. An overview of nosocomial infections including the role of the microbiology laboratory. Clin Microbiol Rev 1993; 6: 428-42.

7. Gesu GP, Marchetti F, Piccoli L, Cavallero A. Levofloxacin and ciprofloxacin in vitro activities against 4003 clinical bacterial collected in italian laboratories. Antimicrob Agents Chemother 2003; 47: 816-9.

8. Girare R. Guide technique d'hygiène hospitalierè. Alger,Institut de la Santè publique et Lyon, Fondation Marace Merieux, 1990 (17 CAP).

9. Hermann M. Vaudaux PE, Pittet D. Fibronectin, fibrinogen and laminin act as mediator of adherence of clinical staphylococcual isolates to foreing material. J Infect Dis 1988; 158: 653-702.

10. Jaber B. Bacterial infections in hemodialysis patients: pathogenesis and prevention.: Kidney International 2005; 67: 2508-19.

11. Maki DG, Goldman DA, Rhames FS. Infection control in intravenous therapy.Ann Int Med 1973; 79: 867-87.

12. Maki DG, Cobb I, Garman JK, et al. An attachable silver-impregnant cuff for prevention of infection with central venous catheters. A prospective randomised multi-center trial. Am J Med 1988; 85: 307-14.

13. Maki DG. Pathogenesis, prevention, and management of infections due to intravascular devices used for infusion therapy. In: Bisno A, Valdvogel F. ed. Infections associated with indwelling medical devices, American Society for Microbiology, Washington D.C.: 1989; 161-77.

14. Moro ML. Infezioni ospedaliere: prevenzione e con- trollo Ed. Centro Scientifico Editori 1993

15. O'Grady NP, Alexander M, Dellinger EP, et al. Guidelines for the prevention of intravascular catheter-related infections. Clin Infect Dis 2002; 35: 1281-307.

16. Paragiuodaki M, Stamouli F, Anastassiou D, Dimitracopoulos G, Spilopoulou I. Intravenous catheter infections associated with bacteraemia: a 2year study in a University hospital. Cli Microbiol Infect 2004; 10: 431-5.

17. Pascual A. Pathogenesis of catheter-related infections: lessons for new design. Clin Microbiol Infect 2002; 8; 256-64.

18. Pelletier SJ, Crabtree TD, Gleason TG, Pruett TL, Sawyer LG. Bacteremia associated with central venous catheter infection is not an indipendent predictor of outcomes. J Am Coll Surg. 2000; 190: 671-80.

19. Raad I. Intravascular catheter-associated infections. Lancet 1998; 351: 893-8.

20. Vaudaux P, Francois P, Lew DP, Waldvogel FA, Bisno AL Eds. Infection associated with indwelling medical devices, 3rd edn. Washington DC A.S.M. 2000; 1-26.

Giacomo Fortina

A.O. Maggiore della Carità

U.O.A. di Microbiologia

C.so Mazzini, 18 - 28100 Novara

Tel. 03213733375 - Fax 03213733240 giacomo.fortina@maggioreosp.novara.it 